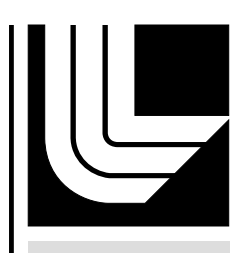

LA WRENCE LIVERMORE NATIONAL LABORATORY

ALTERNATIVE METHODS FOR TRITIUM RECOVERY FROM THE LIFELITHIUM BLANKET: FY13 FINAL REPORT

T. Bandhauer, J. Adler

October 29, 2013 
This document was prepared as an account of work sponsored by an agency of the United States government. Neither the United States government nor Lawrence Livermore National Security, LLC, nor any of their employees makes any warranty, expressed or implied, or assumes any legal liability or responsibility for the accuracy, completeness, or usefulness of any information, apparatus, product, or process disclosed, or represents that its use would not infringe privately owned rights. Reference herein to any specific commercial product, process, or service by trade name, trademark, manufacturer, or otherwise does not necessarily constitute or imply its endorsement, recommendation, or favoring by the United States government or Lawrence Livermore National Security, LLC. The views and opinions of authors expressed herein do not necessarily state or reflect those of the United States government or Lawrence Livermore National Security, LLC, and shall not be used for advertising or product endorsement purposes.

This work performed under the auspices of the U.S. Department of Energy by Lawrence Livermore National Laboratory under Contract DE-AC52-07NA27344. 


\title{
ALTERNATIVE METHODS FOR TRITIUM RECOVERY FROM THE LIFE LITHIUM BLANKET: FY13 FINAL REPORT
}

\author{
Dr. Todd M. Bandhauer and Jonas Adler
}

Interdisciplinary Thermal Science Laboratory, Department of Mechanical Engineering, Colorado State University

\section{EXECUTIVE SUMMARY}

One of the critical components for the Laser Inertial Fusion Energy (LIFE) power plant is the recovery of tritium from the reactor lithium cooling blanket. In the process proposed by Maroni $[1,2]$, high temperature halide salts extract tritium from the lithium using a high-speed centrifugal contactor. Because of high-temperature fluid mixture is very corrosive, this system is not practical. The LIFE team has proposed an alternative process that utilizes a halide-free salt (e.g., $\mathrm{LiOH}$ ) with a non-centrifuge separation process to extract tritium from the lithium cooling blanket, thus eliminating the need for high-temperature, halide corrosion-resistant materials. To facilitate the design of the contactor and separator, Colorado State University (CSU) was subcontracted by LLNL to conduct the following initial steps in the design process during FY13:

- Develop preliminary designs for the separator and Plexiglas models for flow visualization and computational model development

- Select surrogate fluids and flow conditions for the separator

- Select an appropriate flow visualization fluorescent dye for the surrogate tritium solute.

As detailed in the report, CSU has successfully completed all of these tasks. For the design of the actual and surrogate separator, the CSU research team utilized the hydrocyclone design by Colman [9] (Figure 5 and Table 2) for the separation of highly concentrated dispersed oil droplets from water, and used a dimensionless scaling method 
for the initial $\mathrm{Li} / \mathrm{Li}$-salt system separator design. The CSU research team has also selected several surrogate working fluids for the $\mathrm{Li} / \mathrm{Li}$-salt system: oil/water, water/HFE7500, water/R-120, and hexane/water, and HFE7500/hexane. The oil/water system will be used first in the planned experimental facility, and flow conditions are summarized in Table 3 for the surrogate system and the $\mathrm{Li} / \mathrm{Li}$-salt system. Acetone-based fluorescent dyes are available from Carco Industrial Products (e.g., 1044), and, because they are miscible in both non-polar liquids and water, they can be used as a surrogate to mimic tritium mass transport. In addition to mimicking tritium separation, solvent-soluble fluorescent dyes that are immiscible in water (e.g., Keystone Liquid Oil Red HF) can also be utilized to measure local oil concentration through the utilization of a planar laser induce fluorescence system (PLIF). This technique and a particle image velocimetry (PIV) system will be used to validate computation models currently under development.

\section{INTRODUCTION}

The LIFE power plant design uses a liquid lithium blanket for cooling the reactor.

Neutrons produced from the fusion process react with the lithium to produce tritium. Because radioactive tritium, a rare and expensive isotope of hydrogen, is a required fuel for the fusion process, it must be extracted from the lithium cooling blanket and stored to make fuel pellets. In the process proposed by Maroni $[1,2]$, high temperature halide salts extract tritium from the lithium using a high-speed centrifugal contactor. Because of high-temperature fluid mixture is very corrosive, this system is not practical. The LIFE team has proposed an alternative process that utilizes a halide-free salt (e.g., $\mathrm{LiOH}$ ) with a non-centrifugal separation process, thus eliminating the need for high-temperature, halide corrosion-resistant materials. In the proposed method, the one of the phases is dispersed into small droplets within the other phase to maximize surface area to increase 
tritium mass transfer. Once the tritium in transferred into the salt, the two phases are separated. The tritium is then extracted from lithium salt phase through electrolysis, while the pure lithium phase returns to the blanket.

One of the key challenges for this system is the design of the non-centrifuge separator, which LLNL has assigned to CSU. In this current phase of the project, the CSU research team was required to conduct the following initial steps in the design process:

- Develop preliminary designs for the separator and Plexiglas models for flow visualization and computational model development

- Select surrogate fluids and flow conditions for the separator

- Select an appropriate flow visualization fluorescent dye for the surrogate tritium solute

As discussed in this report, CSU has completed all of these steps. The outline of the report is as follows. First, a discussion of the fundamental operation of the proposed non-centrifugal separator, a hydrocyclone, is discussed. This is followed by a description of the initial hydrocyclone design and methodology for scaling geometry and flow conditions for surrogate fluid and $\mathrm{Li} / \mathrm{Li}$-salt systems. In this section, alternatives to oil/water surrogate systems are discussed. Finally, the report summarizes details of the anticipated experiments, including the selection of appropriate fluorescent dyes for mimicking tritium mass transport and the verification of computational models.

\section{REVIEW OF PRIOR HYDROCYCLONE RESEARCH}

Hydrocyclones are frequently used to separate particles and fluid droplets suspended in another fluid. Because the lithium and lithium salt will be in the liquid 
phase, the brief review presented here will focus on hydrocyclones that separate immiscible liquids of two different densities. The most common application for liquid/liquid hydrocyclone separators are the de-oiling of water supplies in the oil and gas industry, and most of the relevant literature focuses on this specific application. In the following sections, the basic fluid mechanics of hydrocyclones is presented, followed by a discussion of applicable prior work, including both computational and experimental investigations. Because the concentration of the lithium in the lithium salt is expected to be high, particular attention is paid to studies that investigate high feed concentrations of separated fluid.

\section{Hydrocyclone Operation}

As shown in Figure 1, the process fluid mixture enters tangential to the cylindrical portion of the hydrocyclone, and exits perpendicular to the inlet along the axis through tubes on the cylinder (overflow) and at the exit of the conical section (underflow). The

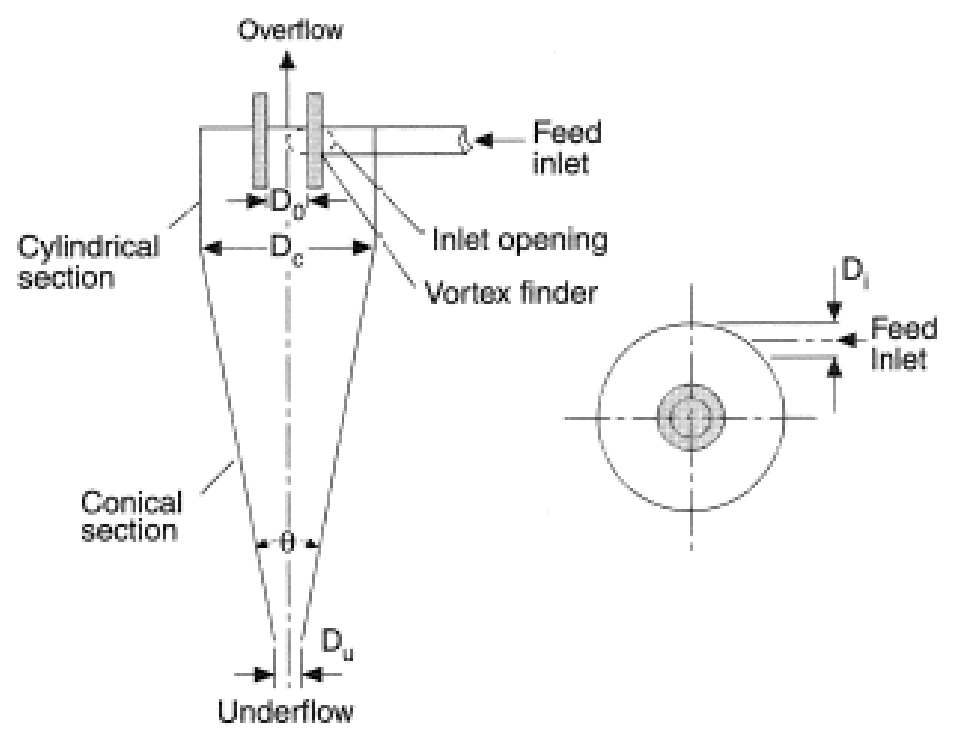

Figure 1: Representative Hydrocyclone Geometry [3] 
high inlet feed flow and the shape of the hydrocyclone produce high azimuthal velocities (up to $20 \mathrm{~m} \mathrm{~s}^{-1}$ ), which imparts a passive centrifugal force field onto the flow and yields an inward radial velocity (usually less than $0.1 \mathrm{~m} \mathrm{~s}^{-1}$ ). In principle, the phase with the higher density migrates towards the outer wall of the hydrocyclone, and the resulting centripetal force moves the lower density phase toward the hydrocyclone axis.

Two axial vortices are produced in the hydrocyclone: a primary vortex rotating near the wall and flowing toward the underflow, and a central secondary vortex flowing towards the overflow. As a consequence of the counterflowing vortices, a shear plane exists where the axial velocity is zero: the Locus of Zero Vertical Velocity (LZVV). In theory, there is a particle (or droplet) size that will orbit precisely at the LZVV and will, therefore, have an equal chance of entering the primary and the secondary vortex. This particle size is known as the "cut size". As the characteristic cut size reduces, the separation efficiency of the hydrocyclone increases due to the concomitant increase in centripetal separation force.

In the subsequent two sections, prior computational and experimental investigations relevant to the dual liquid $\mathrm{Li} / \mathrm{Li}$-salt phase separation process are discussed. It is anticipated that the Li mass fraction of the hydrocyclone inlet will be relatively high (i.e., near 0.5). Therefore, specific attention is paid to the relatively few studies that investigate high inlet feed concentrations (i.e., > 10\%).

\section{Relevant Computational Investigations}

Several approaches have been employed to model the multiphase flow inside hydrocyclones. However, the number of studies relevant to $\mathrm{Li} / \mathrm{Li}$-salt separations (i.e., high feed concentrations or the use of molten metals) is extremely limited. Huang [6] simulated the performance of a liquid/liquid hydrocyclone design $[9,10]$ (Figure 2) at 


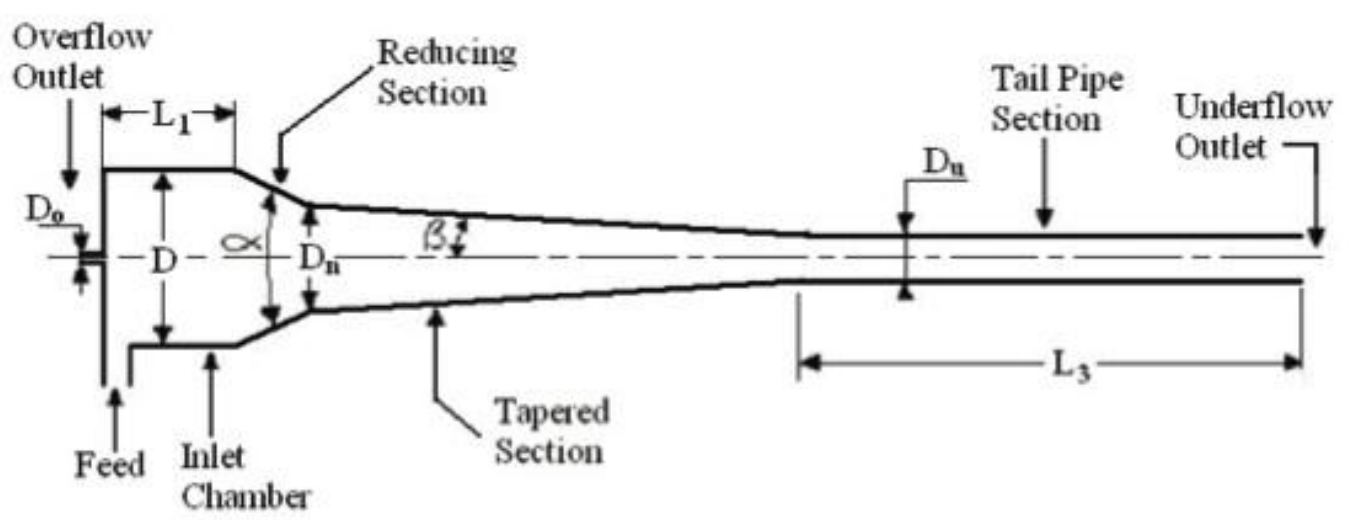

Figure 2: Colman's Hydrocyclone Design [6]

high volume fractions $(\geq 10 \%)$ using the Euler-Euler method with a Reynolds-stress turbulence model (RSM). The Euler-Euler approach assumes that the phases are interpenetrating fluids with no distinguishable interface, and coupling between the two phases is determined through the use of momentum exchange coefficients [11]. Although this method can handle high volume fractions of oil in the inlet feed, the simulation method was validated with experimental data collected on a hydrocyclone with only 5\% inlet oil volume fraction (using polypropylene beads) [10]. At this condition the simulation achieves reasonable agreement with the data: $81.2 \%$ vs. $78.7 \%$ separation efficiency for the simulation and experiment, respectively. However, the CSU research team could not reproduce Huang's numerical efficiency calculation based on the data given in the paper.

Liu et al. [8] also simulated the performance of a purely cylindrical hydrocyclone (i.e., no conical section) using the Euler-Euler approach with the RSM turbulence model, which was were compared to data collected at an oil feed concentration of $17 \%$ (assuming homogeneous flow) at various flow split ratios (Figure 3). The simulation predicted the separation efficiency data to within a maximum relative error of $2.7 \%$, 


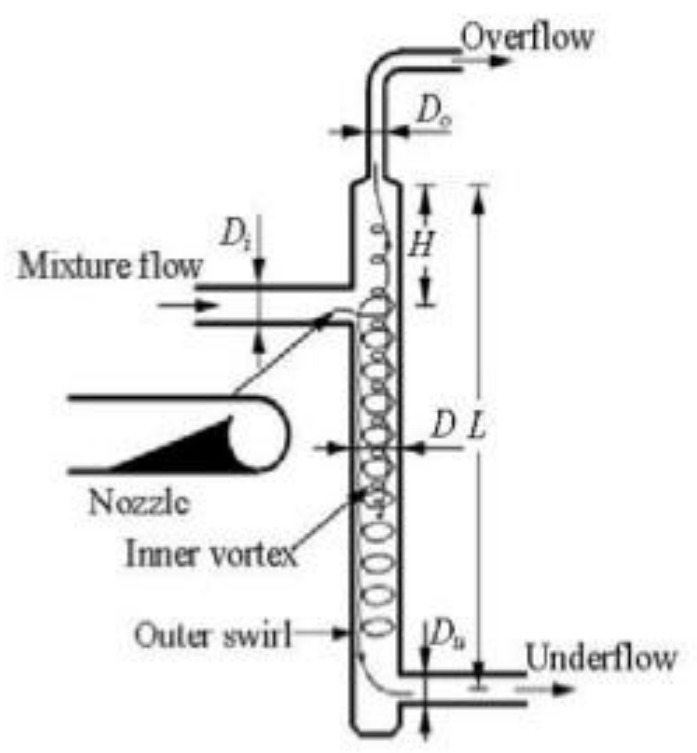

\section{Figure 4: Cylindrical Hydrocyclone Design [8]}

achieving the best accuracy at higher flow-split ratios (i.e., ratio of overflow to inlet flow rates) (Figure 4).

Turchin et al. [12] used the Euler-Lagrangian method with a renormalized group turbulence model (RNG, $k-\varepsilon$ ) to simulate a cyclone designed to remove impurities from molten aluminum. In this method, the spherical particles are assumed to have negligible

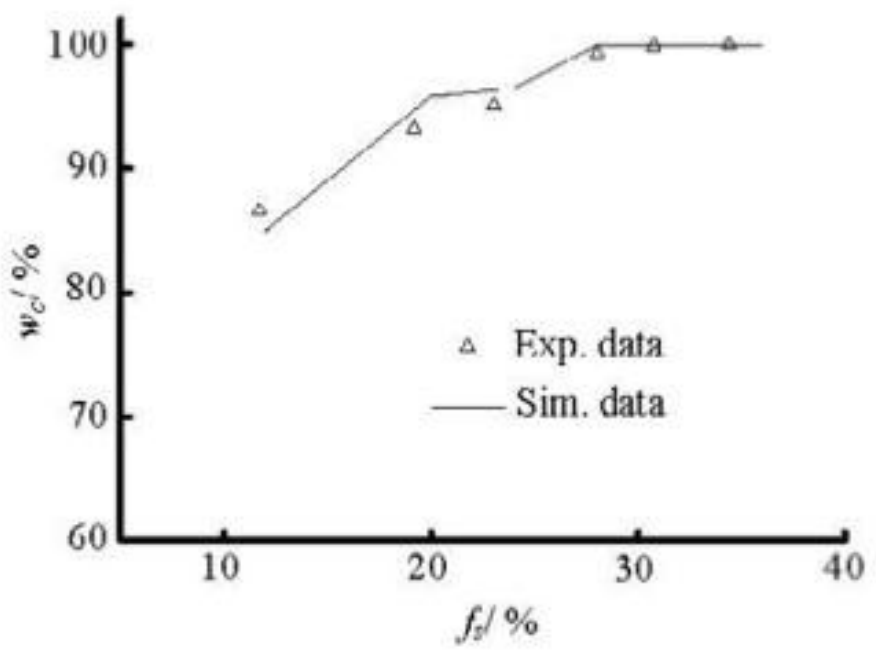

Figure 3: Experimental vs. Simulation Efficiency for the Cylindrical Hydrocyclone Design of Liu et al. [8] 
impact on the flow field. The momentum and continuity equations are used to predict the molten aluminum flow field first, then the particle transport equation is solved assuming that the particles do not influence each other. As a result, the Euler-Lagrangian method is valid only for low particle concentrations. Their results showed axial and tangential velocities that agreed well with simulation data of Gupta and Kumar [13]. However, the authors noted that additional experimentation is required to ensure that the simulation methodology is valid.

\section{Relevant Experimental Investigations}

The majority of experimental investigations on oil/water hydrocyclone separators are for low inlet oil concentrations, and few experimental studies exist for oil/water hydrocyclones at higher concentrations of the dispersed phase. Some of these studies are reviewed here.

In addition to their numerical analysis, Liu et al. [8] also performed experiments on their purely cylindrical hydrocyclone design (Figure 3). They studied the effect of flow split-ratio and Reynolds number on separation efficiency. The water flow-rates were varied from $2.5 \mathrm{~m}^{3} \mathrm{~h}^{-1}$ to $8.75 \mathrm{~m}^{3} \mathrm{~h}^{-1}$, while the oil volume fraction in the inlet feed was varied from $4 \%$ to $30 \%$. The showed that an increased flow-split ratio significantly increased the separation efficiency, approaching a 100\% water mixture in the underflow. In addition, as the flow-split ratio was initially increased, the oil concentration in the overflow also increased. However, the oil concentration in the overflow reached a maximum value and subsequently decreased as the flow-split was increased further. The inlet flow rate also affected the separation efficiency, but the effect was small compared to the influence of flow split-ratio. 
Colman et al. [10] investigated three oil/water hydrocyclone designs using both oil and light solid particles (polypropylene) as a surrogate for oil. In their experiments, the oil concentrations did not exceed 3\%, and the flow-split ratio was held at 10\% for the majority of tests. In contrast to Liu et al. [8], they showed that flow-split ratio had little effect on separation efficiency until the ratio was reduced to approximately the same value as the inlet oil concentration. In addition, as the inlet flow rate increased, the separation efficiency increased for two of the three designs. This is not surprising because the high flow rate is necessary to create high centripetal forces required for separation. However, in their third design, the separation efficiency reached a maximum and subsequently decreased as the flow rate increased. This was attributed to the breakup of oil droplets caused by high-shear at the high flow rates, which was verified by comparing the droplet size distributions at the inlet and outlets.

They also showed that smaller hydrocyclones can achieve greater efficiency at a reduced less pressure drop relative to larger designs. This suggests that using multiple small hydrocyclones instead of a single larger unit would be advantageous. For example, they placed a smaller hydrocyclone downstream of a larger separator to further de-oil the treated water. This combination of hydrocyclones increased efficiency from $82 \%$ to $95 \%$ and from $96 \%$ to $99 \%$ for 40 and 85 micron droplets, respectively.

\section{Summary Prior Work}

In summary, very few prior experimental and numerical investigations on hydrocyclones are relevant to the proposed $\mathrm{Li} / \mathrm{Li}$-salt tritium separation system for the LIFE power plant. Most of the prior work is focused on the purification of water with low oil volume concentrations. Furthermore, many of the papers that do investigate higher oil concentrations omit important geometric and flow details. For example, although Colman 
et al. [10] provide valuable insight on hydrocyclone design and operation, they fail to include the actual dimensions of the three hydrocyclones tested. In addition, as noted by Huang [6], Colman's prior work purportedly included experiments on oil volume concentrations (using polypropylene particles) as high as $30 \%$, but it is unclear how well the Euler-Euler modeling technique used by Huang can sufficiently model these conditions because the results presented are for a lower inlet concentration (10\%). Moreover, these polypropylene particles cannot agglomerate, and may not be representative of an oil and water system. Thus, there is a need to understand the effectiveness of modeling approaches for liquid/liquid hydrocyclones using experimental data gathered on real fluids. In the next section, the dimensionless analysis approach for designing the $\mathrm{Li} / \mathrm{Li}$-salt hydrocyclone separator is described. This is followed by a description of the initial hydrocyclone design for future experiments and simulations using oil/water and other surrogate fluid mixtures, and the anticipated scaled Li/Li-salt separator system design.

\section{HYDROCYCLONE DESIGN APPROACH}

The iterative approach for designing the Li/Li-salt separator is generally comprised of four primary steps: (1) initial design selection and modeling, (2) numerical model validations, (3) dimensionless scaling to model the $\mathrm{Li} / \mathrm{Li}$-salt system, and (4) final design selection. For the first step, an existing hydrocyclone design used for oil/water separation is selected and subsequently modeled for high inlet oil concentrations. Thereafter, the numerical modeling approach will be validated using relevant literature and experimental data gathered on a system the CSU research team will fabricate. Once the model is verified, the flow and geometry required to separate Li from the Li-salt will be determined via scaling from the surrogate fluid system to the Li/Li-salt systems. 
Finally, the design can be iterated upon using the process flow requirements to meet the require tritium inventory limits.

Because it is used to appropriately scale the flow and geometry for various fluid combinations, the non-dimensional analysis will also be critical for guiding the initial design and modeling of the hydrocyclone. The preliminary non-dimensional analysis is presented here. During the modeling and experimental efforts, these non-dimensional numbers may be modified as appropriate. These parameters will also be used to develop correlations to predict separation efficiency of different geometry and/or flow conditions to minimize the number of required high fidelity computer simulations.

The Buckingham-Pi theorem [14] states that the number of dimensionless variables used to describe a system is equal to the number of variables minus the number of primary dimensions. These dimensionless variables can be used to scale a given system. For a hydrocyclone, there are 3 primary dimensions, and 18 key variables identified by the CSU research team for the Colman design (Figure 5):

- Geometry: square inlet width/height $\left(D_{\mathrm{i}}\right)$, overflow diameter $\left(D_{\mathrm{o}}\right)$, underflow diameter $\left(D_{\mathrm{u}}\right)$, major cyclone diameter $\left(D_{\mathrm{c}}\right)$, minor cyclone diameter $\left(D_{\mathrm{r}}\right)$, hydrocyclone lengths $\left(L_{1}, L_{2}, L_{3}, L_{4}\right)$

- Flow: Bulk inlet velocity $\left(V_{\mathrm{i}}\right)$, inlet mass flow rates $\left(\dot{m}_{\mathrm{i}, 1}, \dot{m}_{\mathrm{i}, 2}\right)$, non-dispersed phase overflow mass flow rate $\left(\dot{m}_{\mathrm{o}, 2}\right), 50 \%$ separation cut size $\left(d_{50}\right)$

- Properties: density of each fluid $\left(\rho_{1}, \rho_{2}\right)$, viscosity of each fluid $\left(\mu_{1}, \mu_{2}\right)$

This results in a total of 15 pi terms. (The reader will note that the $50 \%$ separation cut size yields equal dispersed phase mass flow rates in the underflow and the overflow. Continuity can be used to directly calculate these dispersed mass flow rates, thus 
eliminating the need for an extra dimensionless variable.) However, the required dimensionless variables and their mathematical relationship are not specified by the Buckingham-Pi theorem. The development of these variables and their functional relationships require a combination of physical reasoning and experimental evidence.

Castilho and Medronho [15] proposed that the most important non-dimensionless parameters for the prediction of hydrocyclone separation performance are the Stokes and Reynolds numbers based on the inlet velocity defined as follows:

$$
\begin{gathered}
S t k_{50}=\frac{\left(\rho_{1}-\rho_{2}\right) V_{\mathrm{i}}\left(d_{50}\right)^{2}}{18 \mu_{\mathrm{eff}} D_{\mathrm{c}}} \\
\operatorname{Re}_{\mathrm{i}}=\frac{\rho_{\mathrm{eff}, \mathrm{i}} V_{\mathrm{i}} D_{\mathrm{c}}}{\mu_{\mathrm{eff}, \mathrm{i}}}
\end{gathered}
$$

As with most low dispersed phase concentration investigations, the effective density ( $\left.\rho_{\text {eff }}\right)$ and viscosity $\left(\mu_{\mathrm{eff}}\right)$ are the non-dispersed fluid properties. For high dispersed-phase concentrations, the CSU research team believes that the density and viscosity of both phases must be included to achieve inlet Reynolds number similarity. Furthermore, the fluid flow conditions at the underflow and overflow are also important, and the proposed Reynolds numbers at each of these locations are as follows:

$$
\begin{gathered}
\operatorname{Re}_{\mathrm{o}}=\frac{4\left(\dot{m}_{\mathrm{o}, 1}+\dot{m}_{\mathrm{o}, 2}\right)}{\pi D_{\mathrm{o}} \mu_{\mathrm{eff}, \mathrm{o}}} \\
\operatorname{Re}_{\mathrm{u}}=\frac{4\left[\left(\dot{m}_{\mathrm{i}, 1}+\dot{m}_{\mathrm{i}, 2}\right)-\left(\dot{m}_{\mathrm{o}, 1}+\dot{m}_{\mathrm{o}, 2}\right)\right]}{\pi D_{\mathrm{u}} \mu_{\mathrm{eff}, \mathrm{u}}}
\end{gathered}
$$

It can be shown that the overflow mass flow rate for the dispersed phase is calculated via conservation of mass from the three known flow rates and assuming the 50\% separation (i.e., $d_{50}$ ); thus, it does not introduce an additional variable. Because the dispersed phase 
consists of small droplets for the $\mathrm{Li} / \mathrm{Li}$-salt system to enhance mass transfer, a homogeneous flow at the inlet and outlets is a valid assumption. Thus, the effective density and viscosity values are calculated as follows:

$$
\begin{aligned}
& \frac{1}{\rho_{\mathrm{eff}}}=\frac{M R}{\rho_{1}}+\frac{1-M R}{\rho_{2}} \\
& \frac{1}{\mu_{\mathrm{eff}}}=\frac{M R}{\mu_{1}}+\frac{1-M R}{\mu_{2}}
\end{aligned}
$$

where:

$$
M R=\frac{\dot{m}_{1}}{\dot{m}_{1}+\dot{m}_{2}}
$$

The final flow-based dimensionless number is the flow split, which is defined as follows:

$$
F S=\frac{\dot{m}_{\mathrm{o}, 1}+\dot{m}_{\mathrm{o}, 2}}{\dot{m}_{\mathrm{i}, 1}+\dot{m}_{\mathrm{i}, 2}}
$$

The analysis thus far accounts for 7 dimensionless parameters: $S t k_{50}, R e_{\mathrm{i}}, R e_{\mathrm{o}}, R e_{\mathrm{u}}, M R_{\mathrm{i}}$, $M R_{\mathrm{o}}$, and $F S .\left(M R_{\mathrm{u}}\right.$ can be calculated directly from $M R_{\mathrm{o}}, M R_{\mathrm{i}}$, and the mass flow rates at the inlet and overflow.) The remaining 8 dimensionless parameters are assumed to be simple geometric relationships:

$$
\frac{D_{\mathrm{i}}}{D_{\mathrm{c}}}, \frac{D_{\mathrm{u}}}{D_{\mathrm{c}}}, \frac{D_{\mathrm{o}}}{D_{\mathrm{c}}}, \frac{D_{\mathrm{r}}}{D_{\mathrm{c}}}, \frac{L_{3}}{D_{\mathrm{c}}}, \frac{L_{1}}{L_{3}}, \frac{L_{2}}{L_{3}}, \frac{L_{4}}{L_{3}}
$$

Several investigators have utilized similar non-dimensional parameters to predict performance. For example, Overcamp and Scarlett [16] investigated the relationship between the Stokes number, Reynolds number, and cyclone geometry. Utilizing data from multiple sources, they concluded that the Stokes and Reynolds numbers are inversely correlated in most cases, but suggested further experimentation to verify this relationship. The functional relationship between all 15 non-dimensional terms suggested 


\section{Table 1: Properties for Lithium and Representative Surrogate Fluids and}

Lithium Salts

\begin{tabular}{|l|c|c|c|c|c|}
\hline \multicolumn{1}{|c|}{ Item } & Oil & Water & $\mathbf{L i}$ & $\mathbf{L i O H}$ & $\mathbf{L i}_{2} \mathbf{C o}_{3}$ \\
\hline $\mathrm{T}\left[\mathrm{o}^{\mathrm{C}}\right]$ & 20 & 20 & 500 & 500 & 777 \\
\hline$\rho^{\left[\mathrm{kg} / \mathrm{m}^{3}\right]}$ & 890 & 998 & 457 & 1460 & 1811 \\
\hline$\mu[\mathrm{cP}]$ & 3.32 & 1.00 & 0.24 & $?$ & 4.64 \\
\hline
\end{tabular}

by the CSU research team will be developed using a combination of experimental and computational analysis. In the next section, the procedure for scaling designs for different fluid combinations using these dimensionless variables is discussed, including a discussion on the availability of lithium salt properties.

\section{HYDROCYCLONE DESIGN}

Liquid/liquid hydrocyclones are primarily used for water de-oiling, and the vast majority of available computational and experimental studies focus on this application. However, very few studies investigate the effect of high dispersed phase concentration. Therefore, the CSU research team plans to use a de-oiling hydrocyclone design to verify the planned modeling effort, then to investigate the impact of fluid properties using both a $\mathrm{Li} / \mathrm{Li}$-salt and other surrogate fluid systems. In the following sections, the availability of appropriate $\mathrm{Li} / \mathrm{Li}$-salt properties, the scaling methodology, and the initial designs for the oil/water and $\mathrm{Li} / \mathrm{Li}$-salt system are summarized. In addition, some alternatives surrogate fluids are discussed.

\section{Fluid Properties}

To scale the initial oil/water system design to the Li/Li-salt system, accurate properties of all fluids at the necessary temperatures are required. Table 1 summarizes the available fluid properties for oil, water, lithium, and candidate lithium salts $(\mathrm{LiOH}$ and $\mathrm{Li}_{2} \mathrm{CO}_{3}$ ). The temperature dependent formulae for density and viscosity of lithium and 
lithium carbonate are also available $[17,18]$. The liquid viscosity for $\mathrm{LiOH}$ is not available in the literature, which may necessitate experiments to measure it. Special equipment is required for the viscosity due to the elevated temperature of molten $\mathrm{LiOH}$ $\left(T_{\text {melt }}=471^{\circ} \mathrm{C}\right.$ ). Quotes were obtained by the CSU team from two companies (Dynisco and Malvern) for high temperature rheometers. The Dynisco LCR7001 rheometer and high temperature oven can measure fluid viscosity up to $500^{\circ} \mathrm{C}$ and costs approximately \$53K. Malvern's Bohlin Gemini HR Nano rheometer (with an optional high temperature oven) can measure fluid viscosity up to $550^{\circ} \mathrm{C}$, and costs slightly more $(\$ 70-80 \mathrm{~K})$. However, the equipment compatibility of $\mathrm{LiOH}$ with systems is unknown, and requires further exploration.

As an alternative to conducting experiment at LLNL or CSU, the viscosity of $\mathrm{LiOH}$ can be measured by a third party. Dynalene can measure fluid viscosity at temperatures up to $600^{\circ} \mathrm{C}$ ( $\$ 500$ per sample). Additionally, Argonne National Laboratory [19] has used an ultrasonic method to measure of viscosity and density of nuclear waste. However, in both cases, compatibility of $\mathrm{LiOH}$ with these systems has yet to be verified.

\section{Scaling Methodology}

Once the fluid properties and the performance are characterized for an oil/water system, the hydrocyclone can be scaled using the non-dimensional numbers described previously for the Li/Li-salt and surrogate fluid systems. The scaling methodology is described in this section.

After the simulation technique has been verified for a single design, simulations on a scaled design for the Li/Li-salt system will be conducted to verify that the correct non-dimensional parameters were selected, paving the way for additional experimentation to determine the appropriate functional relationship among the 


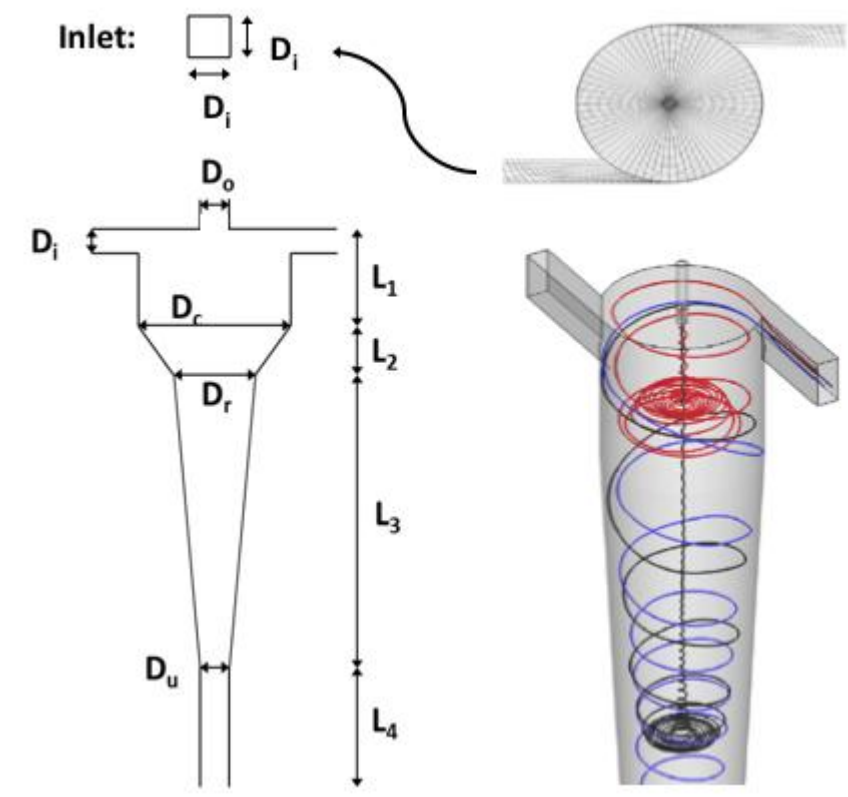

\section{Figure 5: Initial Hydrocyclone Design}

dimensionless terms. To verify that these terms appropriately describe system performance, the CSU research team presumes that the separation capability for both the original and scaled designs are similar, which yield the following assumptions: the 50\% separation particle size $\left(d_{50}\right)$ and the mass ratio for the overflow $\left(M R_{\mathrm{o}}\right)$ are the same for the same flow split ratio $(F S)$ and inlet mass ratio $\left(M R_{\mathrm{i}}\right)$. These conditions should hold true for the same inlet, overflow, and underflow Reynolds numbers and the Stokes number. With these assumptions, Equations 1 through 4 can be solved simultaneously to yield the scaled inlet velocity $\left(V_{\mathrm{i}}\right)$ and major cyclone $\left(D_{\mathrm{c}}\right)$, overflow $\left(D_{\mathrm{o}}\right)$, and underflow $\left(D_{\mathrm{u}}\right)$ diameters. It is also assumed that the remaining geometric parameters $\left(D_{\mathrm{r}}, L_{1}, L_{2}, L_{3}\right.$, and $\left.L_{4}\right)$ can be scale geometrically. These assumptions will be validated through a combination of experiments and simulations. In the following section, the design and flow conditions for the initial surrogate fluid hydrocyclone (i.e, oil/water) and the scaled $\mathrm{Li} / \mathrm{Li}$-salt system are presented. 
Table 2: Geometric Scaling for the Li/Li-salt and Surrogate Fluid Systems

\begin{tabular}{|l|c|c|}
\hline \multicolumn{1}{|c|}{ Dimension } & Water/Oil & $\mathbf{L i} / \mathbf{L i}_{\mathbf{2}} \mathbf{C O}_{\mathbf{3}}$ \\
\hline Cut Size $(\mu \mathrm{m})$ & 20 & 20 \\
\hline $\mathrm{D}_{\mathrm{c}}(\mathrm{cm})$ & 6.00 & 16.74 \\
\hline $\mathrm{D}_{\mathrm{r}}(\mathrm{cm})$ & 3.00 & 8.37 \\
\hline $\mathrm{D}_{\mathrm{o}}(\mathrm{cm})$ & 0.42 & 4.32 \\
\hline $\mathrm{D}_{\mathrm{u}}(\mathrm{cm})$ & 1.50 & 1.33 \\
\hline $\mathrm{D}_{\mathrm{i}}(\mathrm{cm})$ & 0.48 & 1.33 \\
\hline $\mathrm{L}_{1}(\mathrm{~cm})$ & 9.00 & 25.11 \\
\hline $\mathrm{L}_{2}(\mathrm{~cm})$ & 8.50 & 23.72 \\
\hline $\mathrm{L}_{3}(\mathrm{~cm})$ & 64.10 & 178.84 \\
\hline $\mathrm{L}_{4}(\mathrm{~cm})$ & 63.00 & 175.77 \\
\hline
\end{tabular}

\section{Summary of Initial Geometry and Flow Conditions}

Figure 5 shows the geometry for the initial hydrocyclone design. (A representative hydrocyclone design from Saidi et al. [20] is shown for clarity.) The hydrocyclone has two square shaped tangential inlets and dual conical sections. In contrast to other designs, the overflow outlet tube does not extended into the main body of the flow. The impact from eliminating this "vortex finder" is unclear, which can be investigated during the experimental phase of the project.

The dimensions for the surrogate fluid (oil/water) and the $\mathrm{Li} / \mathrm{Li}$-salt system are listed in Table 2, and the flow conditions for each fluid pair are shown in Table 3 . The surrogate fluid design is identical to the design simulated by Huang [6], which will enable the team to compare their results to the numerical model currently under development. The scaled flow and geometry for the Li/Li-salt system was determined from the method described in the previous section. Similar results can be obtained for other surrogate fluids, which may be more reflective of the $\mathrm{Li} / \mathrm{Li}$-salt systems. Potential options for these fluids are described in the next section. 
Table 3: Flow Scaling for the Li/Li-salt and Surrogate Fluid Systems

\begin{tabular}{|l|c|c|}
\hline \multicolumn{1}{|c|}{ Property } & Water/Oil & $\mathbf{L i} / \mathbf{L i}_{2} \mathbf{C O}_{\mathbf{3}}$ \\
\hline Cut Size $(\mu \mathrm{m})$ & 20 & 20 \\
\hline Inlet Velocity $\left(\mathrm{m} \mathrm{s}^{-1}\right)$ & 22 & 12 \\
\hline Inlet Mass Flow Rate $\left(\mathrm{kg} \mathrm{s}^{-1}\right)$ & 1.0 & 3.8 \\
\hline Overflow Mass Flow Rate $\left(\mathrm{kg} \mathrm{s}^{-1}\right)$ & 0.1 & 0.4 \\
\hline Underflow Mass Flow Rate $\left(\mathrm{kg} \mathrm{s}^{-1}\right)$ & 0.9 & 3.4 \\
\hline
\end{tabular}

\section{Potential Alternative Surrogate Fluids}

The best surrogate fluids will closely approximate the relative fluid properties of the lithium and lithium-salt. Unfortunately, oil and water have an inverted viscosity ratio compared lithium and candidate lithium salt $\mathrm{Li}_{2} \mathrm{CO}_{3}$ (Table 4). Two primary options potential exist for selecting different surrogate fluids: (1) a low density/viscosity fluid to pair with water, or (2) a high density/viscosity fluid relative to water. In either case, the fluid must be immiscible in water. Table 5 summarizes a number of low density/viscosity non-polar solvents. All listed solvents are flammable, and have toxicity similar to gasoline. The Engines and Energy Conversion Laboratory at CSU routinely stores and handles similar fuels (Figure 6). For high density/viscosity fluids, a few (relatively) water-immiscible refrigerants that are liquid at atmospheric temperature and pressure are listed in Table 6. These fluids are non-flammable, have low toxicity, and, when mixed with water, their density and viscosity ratios approach Lithium and Lithium-salt. For example, the $\mathrm{Li} / \mathrm{Li}_{2} \mathrm{CO}_{3}$ and Water/HFE7500 have density ratios of 0.3 and 0.6 , respectively, and viscosity ratios of 0.1 and 0.8 , respectively. Although HFE7500 is readily available, it may be difficult to locate a source of R-120, which has relatively high water solubility (450 mg per $100 \mathrm{~g}$ ). A third option combines these two primary options: pair the low density/viscosity solvent with the high density/viscosity refrigerant. For example, combining hexane and water yields density and viscosity ratios of 0.4 and 0.2 , 
Table 4: Density and Viscosity Ratios

\begin{tabular}{|l|c|c|}
\hline \multicolumn{1}{|c|}{ Fluids } & Density Ratio & Viscosity Ratio \\
\hline Oil/Water & 0.9 & 3.3 \\
\hline $\mathrm{Li}^{2} \mathrm{Li}_{2} \mathrm{CO}_{3}$ & 0.3 & 0.1 \\
\hline Hexane/Water & 0.7 & 0.3 \\
\hline Water/HFE7500 & 0.6 & 0.8 \\
\hline Hexane/ HFE7500 & 0.4 & 0.2 \\
\hline
\end{tabular}

respectively. The miscibility of HFE7500 in hexane is unknown, but can be investigated.

In the next section, planned experiments to validate the modeling approach are briefly discussed, with specific attention paid to the selection of the appropriate fluorescent dye for flow visualization.

\section{EXPERIMENTAL CONSIDERATIONS}

As mentioned earlier, experimental data is required to validate computational modeling techniques. In the following sections, the planned flow visualization and measurement experiments (PLIF and PIV) using equipment at CSU are described. One of these methods (PLIF) requires the use of a fluorescent marker. Candidate dyes for this
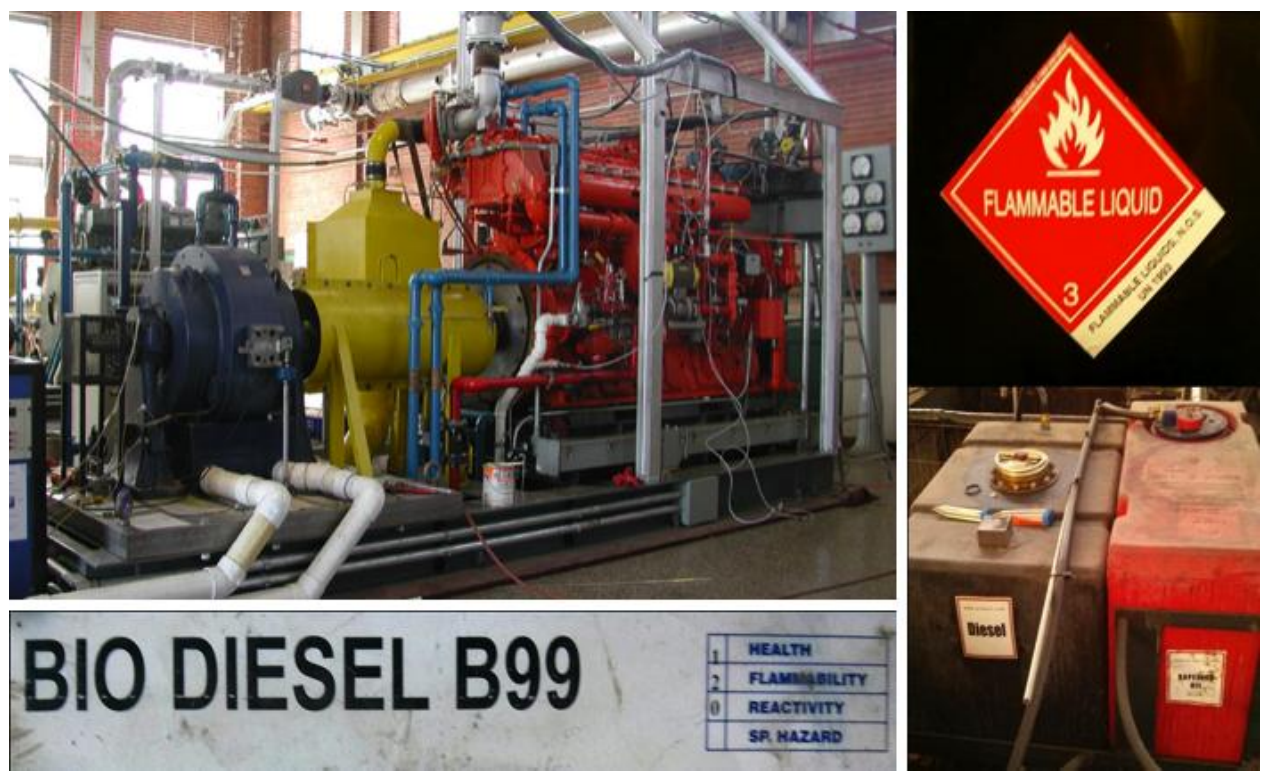

Figure 6: Engines and Energy Conversion Laboratory at CSU 
Table 6: Potential Low Density/Viscosity Surrogate Fluids

\begin{tabular}{|c|c|c|c|c|c|c|c|}
\hline Fluid & $\begin{array}{c}\text { Density } \\
\left(\mathbf{k g ~ m}^{-\mathbf{3}} \mathbf{)}\right.\end{array}$ & $\begin{array}{c}\text { Viscosit } \\
\mathbf{y}(\mathbf{c P})\end{array}$ & $\begin{array}{c}\mathbf{H}_{\mathbf{2}} \mathbf{O} \text { Solub. } \\
(\mathbf{m g} / \mathbf{1 0 0 g})\end{array}$ & $\begin{array}{c}\text { PEL } \\
(\mathbf{p p m})\end{array}$ & \multicolumn{2}{|c|}{$\begin{array}{c}\text { STEL } \\
(\mathbf{p p m})\end{array}$} & \multicolumn{2}{c|}{$\begin{array}{c}\text { Flammabilit } \\
\mathbf{y} \text { Limits (\%) }\end{array}$} \\
\hline Toluene & 862 & 0.36 & 50 & 200 & 150 & 1.3 & 6.8 \\
\hline Butanone & 797 & 0.40 & 25600 & 200 & 300 & 2.0 & 10.0 \\
\hline $\begin{array}{c}\text { Ethyl } \\
\text { Acetate }\end{array}$ & 895 & 0.43 & 8700 & 400 & 400 & 2.0 & 12.0 \\
\hline Hexane & 655 & 0.29 & 14 & 500 & 50 & 1.1 & 7.5 \\
\hline Heptane & 684 & 0.42 & 10 & 500 & 500 & 1.0 & 6.7 \\
\hline $\begin{array}{c}\text { Ethyl } \\
\text { Benzene }\end{array}$ & 874 & 0.60 & 180 & 100 & 125 & 1.4 & 6.7 \\
\hline
\end{tabular}

Table 5: Potential High Density/Viscosity Surrogate Fluids

\begin{tabular}{|c|c|c|c|c|c|c|c|}
\hline Fluid & $\begin{array}{c}\text { Density } \\
\left.\mathbf{( k g ~ m}^{-3}\right)\end{array}$ & $\begin{array}{c}\text { Viscosity } \\
(\mathbf{c P})\end{array}$ & $\begin{array}{c}\text { Density } \\
\text { Ratio }\end{array}$ & $\begin{array}{c}\text { Viscosity } \\
\text { Ratio }\end{array}$ & $\begin{array}{c}\mathbf{H}_{2} \mathbf{O} \\
\text { Solubility } \\
\text { (mg/100g) }\end{array}$ & $\begin{array}{c}\text { PEL } \\
(\mathbf{p p m})\end{array}$ & $\begin{array}{c}\text { Flammability } \\
\text { Limits (\%) }\end{array}$ \\
\hline $\begin{array}{l}\text { HFE } \\
7500\end{array}$ & 1614 & 1.27 & 0.6 & 0.8 & $<0.3$ & $>>100$ & N/A \\
\hline R-120 & 1676 & 2.49 & 0.6 & 0.4 & 490 & None & N/A \\
\hline
\end{tabular}

experiment and for a tritium surrogate are also discussed.

\section{Flow Visualization and Measurement Experiments}

The primary experimental data to be collected includes: axial and azimuthal velocity, inlet and outlet oil droplet size, and local oil volume fraction. For the velocity data, single phase flow experiments are planned using particle image velocimetry (PIV) equipment at CSU (Figures 7 and 8). The system tracks small seedling particles illuminated by a 2-D sheet of laser light to calculate local velocity along the plane. The CSU research team plans measure the axial velocity on a plane perpendicular to the inlet, and rotational velocity field at several locations within the cyclone to verify the applicability of the turbulence model.

To estimate oil droplet size and local concentration, planar laser induced fluorescence (PLIF) will be utilized. PLIF technology consists of laser, optics, camera, and image analysis software (Figure 9 and 10). Similar to PIV, the laser and optics create 


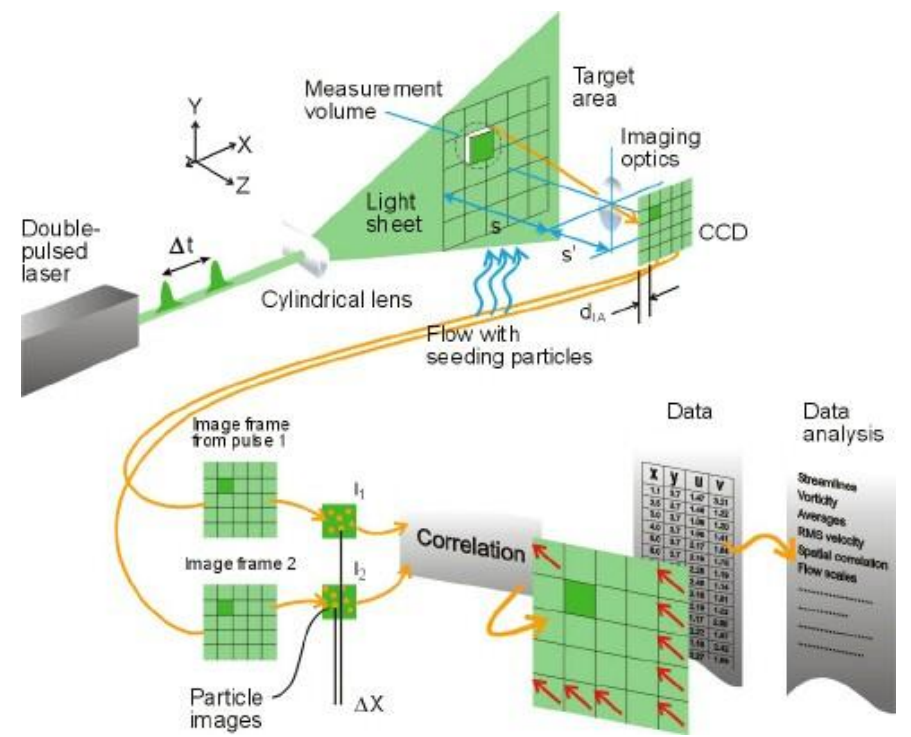

\section{Figure 7: Representative PIV Experimental Setup [4]}

a plane of laser light. A dye imbedded in the fluid fluoresces at the laser wavelength, which is captured by the camera. The image is then analyzed using the accompanying software. CSU has conducted similar experiments on fuel valves for natural gas engines using acetone as the fluorescing species [7]. Dye selection for this project is discussed in the next section.

\section{Fluorescent Dye Selection}

To distinguish between the two phases in the cyclone, it is critical that the dye is miscible in only one of the phases. However, to mimic tritium mass transport, the dye must be soluble in both phases. Therefore, two different dyes are selected: one for numerical modeling validation, and the other as a mass transfer surrogate.

To determine oil and/or solvent concentration and droplet size, a solvent soluble dye can be used. These dyes are inherently insoluble in water, and will, therefore, enable the PLIF system to mark the location of only the selected oil or other non-polar liquid. A candidate solvent soluble dye Keystone Liquid Oil Red HF has been selected for this purpose. For the tritium surrogate, an acetone-based is soluble in both water and non- 

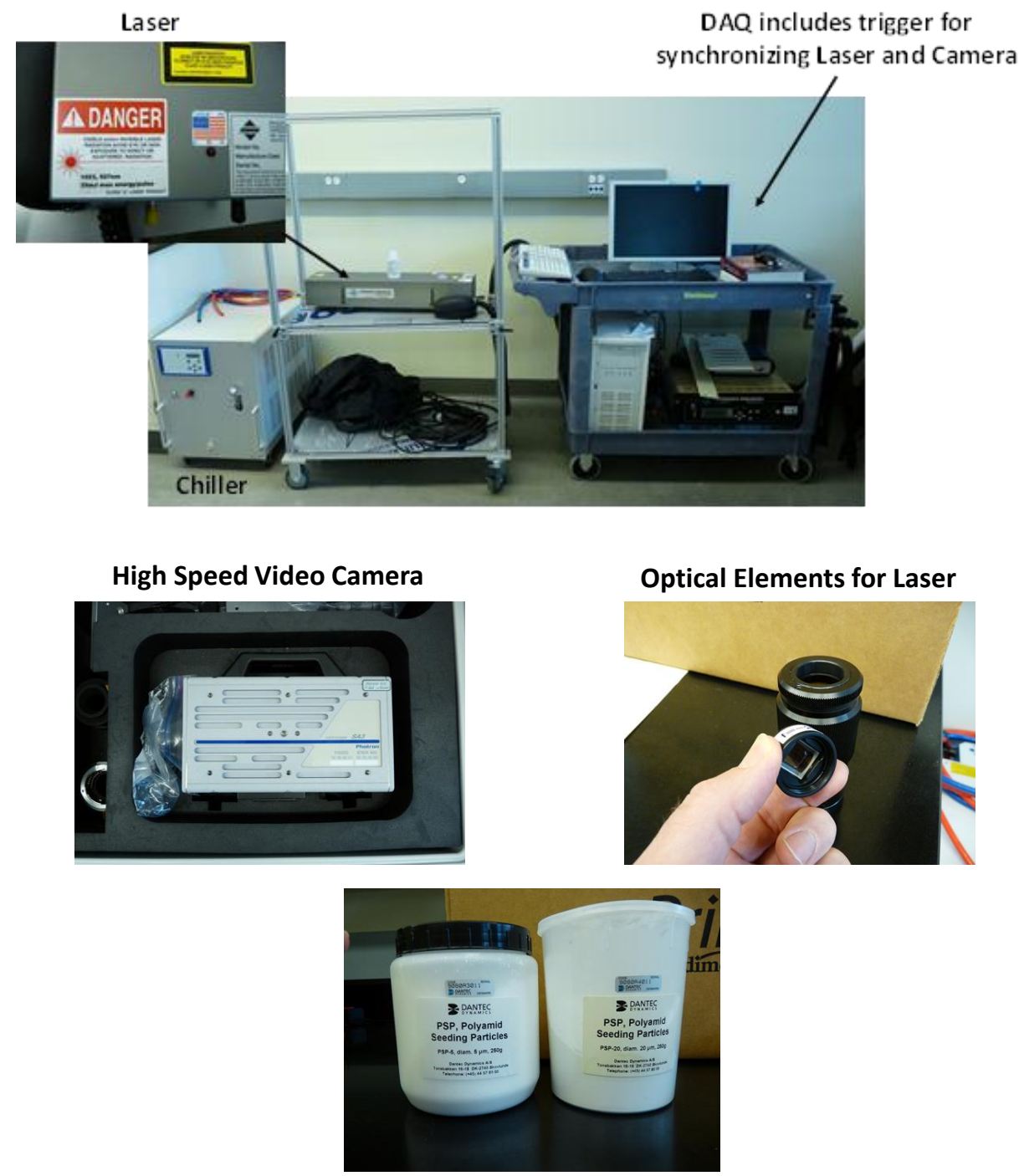

Representative Seedling Particles

\section{Figure 8: PIV Experimental Equipment at CSU}

polar solvents. CARCO Industrial Marking Fluids 1044 is an acetone-based dye used to mark parts, and the CSU research team is planning to use this dye to mimic tritium mass transport. For both candidate dyes, it will be necessary to conduct dye miscibility tests with all candidate surrogate fluids. Several options will be investigated in order to determine the most suitable surrogate fluids and dye combination. 


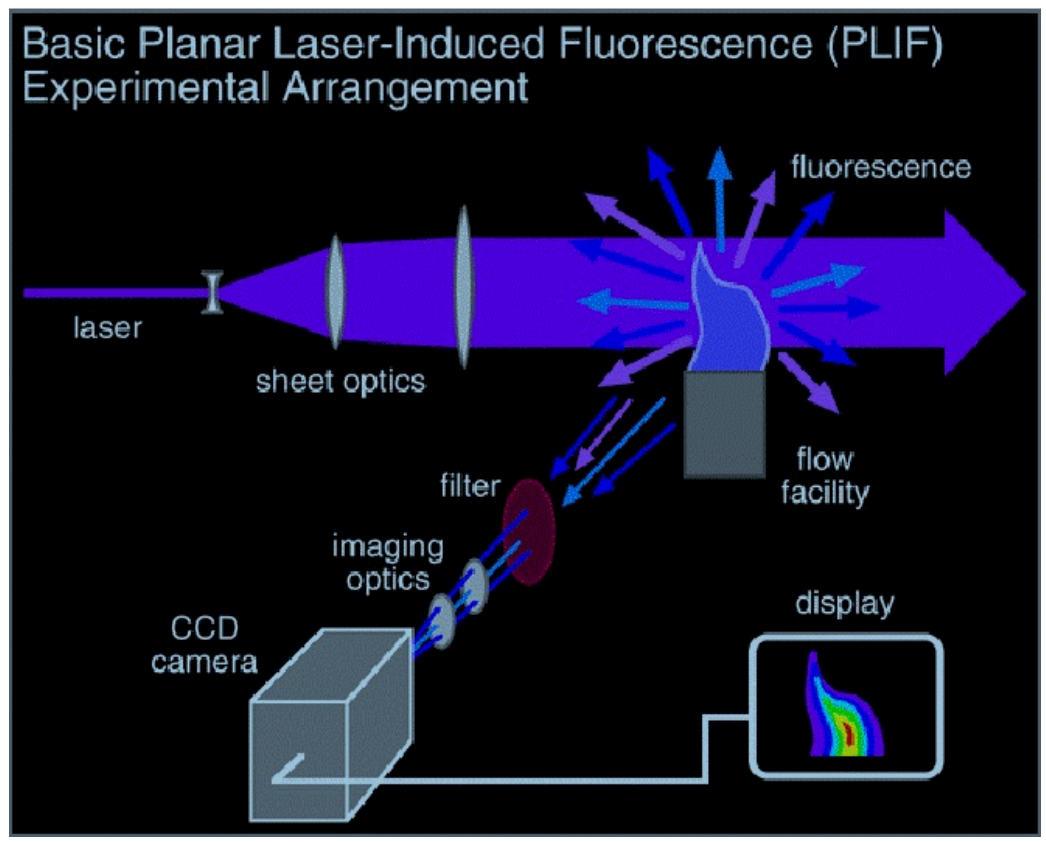

Figure 10: PLIF Experimental Setup [5]
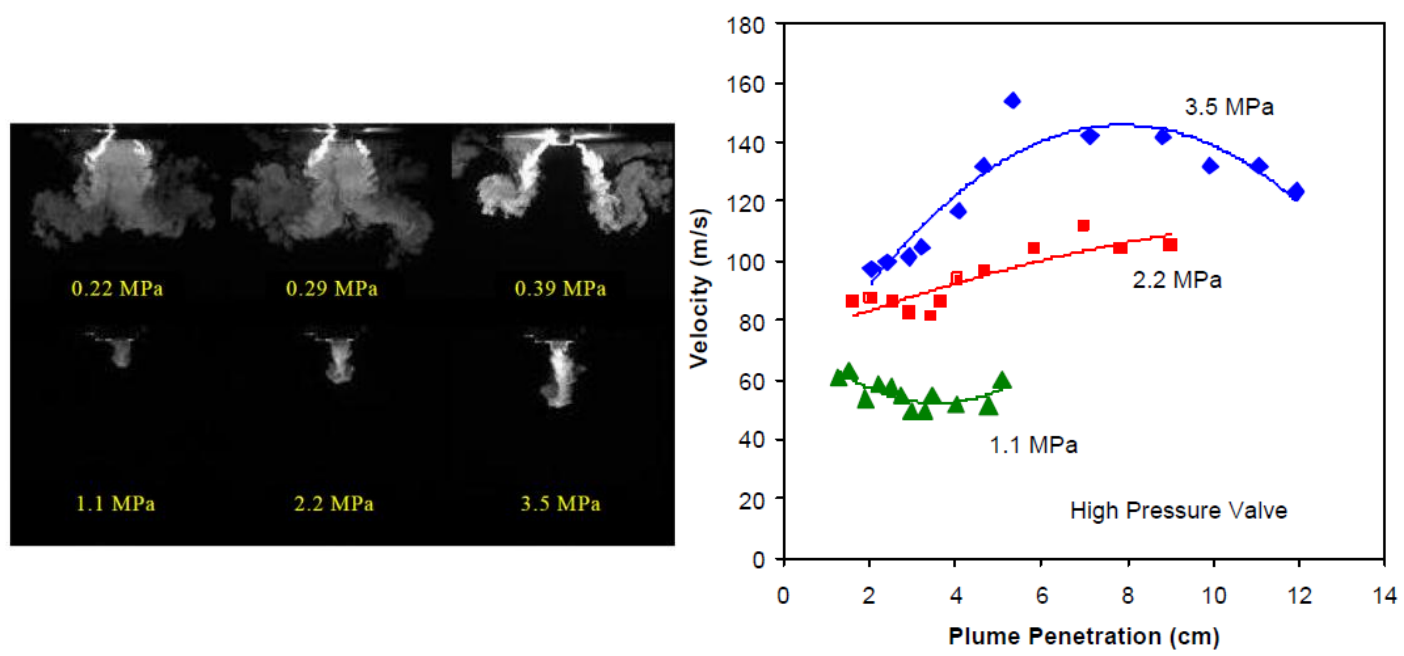

Figure 9: Representative Fuel Valve PLIF Experiments Conducted at CSU Engines and Energy Conversion Lab [7]

\section{SUMMARY}

The CSU research team has developed a preliminary design for the $\mathrm{Li} / \mathrm{Li}$-salt separator and a surrogate model design for flow visualization and computational model development, has selected several candidate surrogate fluids and flow conditions for the separator, and has selected an appropriate flow visualization fluorescent dye for the 
surrogate tritium solute. For the design of the actual and surrogate separator, the CSU research team utilized the hydrocyclone design by Colman et al. $[9,10]$ and simulated by Huang [6] for the separation of highly concentrated dispersed oil droplets from water, and used a dimensionless scaling method for the initial $\mathrm{Li} / \mathrm{Li}$-salt system separator design. There are several candidates for the surrogate working fluids for the $\mathrm{Li} / \mathrm{Li}$-salt system: oil/water, water/HFE7500, water/R-120, hexane/water, and HFE7500/hexane. The oil/water system will be used first in the experimental facility using the same flow conditions as utilized by Huang for the surrogate system to validate the CFD modeling approach, and enable the design of the Li/Li-salt system. Acetone-based fluorescent dyes are available from Carco Industrial Products (e.g., 1044), and, because they are miscible in both non-polar liquids and water, they can be used as a surrogate to mimic tritium mass transport. In addition to mimicking tritium separation, solvent-soluble fluorescent dyes that are immiscible in water (e.g., Keystone Liquid Oil Red HF) can also be utilized to measure local oil concentration through the utilization of a planar laser induce fluorescence system (PLIF). This technique and a particle image velocimetry (PIV) system will be used to validate computation models currently under development. 


\section{REFERENCES}

[1] Maroni, V. A., Wolson, R. D., and Staahl, G. E., 1974, "Some preliminary considerations of a molten-salt extraction process to remove tritium from liquid lithium fusion reactor blankets," First ANS Topical Meeting on the Technology of Controlled Nuclear FusionSan Diego, CA, p. Medium: ED; Size: Pages: 13.

[2] Maroni, V. A., Wolson, R. D., and Staahl, G. E., 1975, "Some preliminary considerations of a molten salt extraction process to remove tritium from liquid lithium fusion reactor blankets," Nucl. Technol., 25(1), pp. 83-91.

[3] Holdrich, R., 2002, Fundamentals of Particle Technology, Midland Information Technology Publishing, Shepshed, Leicestershire, U.K.

[4] Dantec Dynamics, 2008, Measurement Principles of PIV, http://www.dantecdynamics.com/Default.aspx?ID=820.

[5] Velocimetry Knowledge United, 2008, PLIF - Planar Laser Induced Fuorescence, http://www.velocimetry.net/plif_principles.htm.

[6] Huang, S., 2005, "Numerical Simulation of Oil-Water Hydrocyclone Using Reynolds-Stress Model for Eulerian Multiphase Flows," The Canadian Journal of Chemical Engineering, 83, pp. 829-834.

[7] Olsen, D. B., Mastbergen, D. B., and Willson, B. D., 2001, "Planar Laser Induced Fluorescence Imaging of Gas Injection from Fuel Valves for Large Bore Natural Gas Engines," Proceedings ASME-ICE Fall Technical Conference, 37(2), pp. 1724.

[8] Liu, H.-f., Xu, J.-y., Zhang, J., Sun, H.-q., Zhang, J., and Wu, Y.-X., 2012, "Oil/Water Separation in a Liquid-Liquid Cyclindrical Cyclone," Journal of Hydrodynamics, 24(1), pp. 116-123.

[9] Colman, D. A., 1981, "The Hydrocyclone for Separating Light Dispersions," PhD Thesis, University of Southampton, England.

[10] Colman, D. A., Thew, M. T., and Corney, D. R., 1980, "Hydrocyclones for Oil/Water Separation," International Conference on Hydrocyclones, British Hydromechanics Research Association, Cranfield, U.K., pp. 143-166.

[11] FLUENT, 2004, User's Guide 6.2, Chapter 24, FLUENT Inc.

[12] Turchin, A. N., Eskin, D. G., and Katgerman, L., 2008, "Numerical Evaluation of Cyclone Application for Impurities Removal from Molten Aluminum," Metall and Materi Trans B, 39(2), pp. 364-373.

[13] Gupta, A., and Kumar, R., 2007, "Three-dimensional turbulent swirling flow in a cylinder: Experiments and computations," International Journal of Heat and Fluid Flow, 28(2), pp. 249-261.

[14] Munson, B. R., Young, D. F., Okiishi, T. H., and Wade, H., Wade W., 2009, Fundamentals of Fluid Mechanics, John Wiley and Sons, Inc., Hoboken, NJ.

[15] Castilho, L. R., and Medronho, R. A., 2000, "A Simple Procedure for Design and Performance Prediction of Bradley and Rietema Hydrocyclones," Miner. Eng., 13(2), pp. 183-191.

[16] Overcamp, T. J., and Scarlett, S. E., 1993, "Effects of Reynolds Number on the Stokes Number of Cyclones," Aerosol Sci. Technol., 19(3), pp. 362-370.

[17] Janz, G. J., 1968, Molten salts. Volume 1, United States Dept. of Commerce, National Bureau of Standards, Washington, D.C. 
[18] Jeppson, D. W., Ballif, J. L., Yuan, W. W., and Chou, B. E., 1978, "Lithium literature review: lithium's properties and interactions," No. HEDL-TME-78-15.

[19] Sheen, S. H., Chien, H. T., and Raptis, A. C., 1997, Ultrasonic Methods for Measuring Liquid Viscosity and Volume Percent of Solids, Argonne National Laboratory.

[20] Saidi, M., Maddahian, R., Farhanieh, B., and Afshin, H., 2012, "Modeling of Flow Field and Separation Efficiency of a Deoiling Hydrocyclone Using Large Eddy Simulation," Int. J. Miner. Process., 112-113, pp. 84-93. 